\title{
Lack of significant association between mutations of KCNJ10 or FOXI1 and SLC26A4 mutations in pendred syndrome/enlarged vestibular aqueducts
}

\author{
Priya Landa', Ann-Marie Differ ${ }^{1}$, Kaukab Rajput ${ }^{2}$, Lucy Jenkins ${ }^{1}$ and Maria Bitner-Glindzicz ${ }^{1, *^{*}}$
}

\begin{abstract}
Background: Pendred syndrome is a common autosomal recessive disorder causing deafness. Features include sensorineural hearing impairment, goitre, enlarged vestibular aqueducts (EVA) and occasionally Mondini dysplasia. Hearing impairment and EVA may occur in the absence of goitre or thyroid dyshormonogensis in a condition known as non-syndromic EVA. A significant number of patients with Pendred syndrome and non-syndromic EVA show only one mutation in SLC26A4. Two genes, KCNJ10, encoding an inwardly rectifying potassium channel and FOXI1, a transcriptional factor gene, are thought to play a role in the disease phenotypes.
\end{abstract}

Methods: Using Polymerase Chain Reaction and Sanger sequencing, sixty-eight patients with monoallelic mutations of SLC26A4 were tested for mutations in KCNJ10 and FOXI1.

Results: Two variants were observed in the KCNJ10 gene, p.Arg271Cys in three patients and p.Arg18GIn in one patient; only one variant, p.Arg123Trp was observed in the FOXI1 gene in a single patient. Both p.Arg271Cys and p. Arg18Gln are likely to be polymorphisms as judged by their frequency in the general population.

Conclusion: Therefore we found no evidence for a significant association between mutations of KCNJ1O and FOXI1 with SLC26A4. It was also observed that the variant, p.Arg271Cys in KCNJ10, previously thought to have a protective effect against seizure susceptibility, was found in a patient with Pendred syndrome with co-existing epilepsy.

Keywords: Deafness, Enlarged vestibular aqueducts, Epilepsy, EVAS, FOXI1, Hearing impairment, KCNJ10, Pendred syndrome, SLC26A4

\section{Background}

Pendred syndrome (PDS) (OMIM 274600) is one of the most common autosomal recessive disorders of hearing [1,2], characterised by mild to severe sensorineural hearing impairment (SNHL) [2-5] goitre, and inner ear malformations, typically an enlargement of the vestibular aqueduct (EVA) [6]. Some PDS cases can also exhibit Mondini dysplasia which involves the bilateral enlargement of the vestibular aqueducts (EVA) with cochlear hypoplasia [7]. EVA can also be associated with other forms of syndromic and non-syndromic deafness (DFBN4 (OMIM 600791))

\footnotetext{
* Correspondence: maria.bitner@ucl.ac.uk

${ }^{1}$ North East Thames Regional Genetics Service Laboratory, Great Ormond Street Hospital for Children NHS Foundation Trust, 37 Queen Square,York House, London WC1N 3BH, UK

${ }^{3}$ Clinical and Molecular Genetics Unit, UCL Institute of Child Health, 30 Guilford Street, London WC1N 1EH, UK

Full list of author information is available at the end of the article
}

$[8,9]$, particularly non-syndromic enlarged vestibular aqueducts.

Pendred syndrome is the result of malfunction in pendrin, an apical protein anion transporter which mediates chloride $\left(\mathrm{Cl}^{-}\right)$, hydroxide $\left(\mathrm{OH}^{-}\right)$, bicarbonate $\left(\mathrm{HCO}^{-}\right)$ and iodide $\left(\mathrm{I}^{-}\right)$exchange $[10,11]$. Pendrin is expressed in the thyroid and importantly, in the inner ear to maintain the endocochlear potential [5] as well as in the kidney. Dysfunction of ion transport affects iodide organification for thyroid hormone biosynthesis [4] resulting in goitre $[1,3,12]$, which distinguishes it from non-syndromic EVA. However, the majority of patients remain euthyroid [13]. It is now widely acknowledged that mutations in the gene SLC26A4 located on chromosome 7q21-34 [14,15] are involved in the presentation of an EVA/PDS phenotype $[14,16,17]$. Biallelic mutations in the gene SLC26A4 have been shown to cause Pendred syndrome, accounting for 
its autosomal recessive inheritance $[9,14,18]$. However, there is evidence to show that a cohort of patients affected by hearing loss and EVA, have only one mutant allele of SCL26A4; Yang et al. showed that in patients with hearing loss and EVA, in whom thyroid disorder was not used as a clinical criterion, $19 \%$ of siblings had a single mutation in SLC26A4 and $42 \%$ had zero mutations, suggesting that other genetic factors may be involved [19] in the aetiology of their hearing loss. Rendtorff et al., in a clinically wellphenotyped cohort of 109 Scandinavian patients, showed that $10 \%$ had monoallelic mutations. Likely heterogeneity of PDS and non-syndromic EVA has been proposed and investigated, with some suggesting a complex relationship between heterozygosity and the severity of the EVA/PDS phenotype [20].

Two genes, KCNJ1O and FOXI1, have been investigated for their role in the PDS disease spectrum and it has been proposed that digenic mutations in both SLC26A4 and either FOXI1 or KCNJ10 may cause Pendred syndrome [19,21]. The gene KCNJ10 encodes an inwardly rectifying potassium $\left(\mathrm{K}^{+}\right)$channel, namely the Kir4.1 channel, expressed in a wide variety of tissues but most importantly in the case of Pendred syndrome, in the cochlear stria vascularis which maintains the endocochlear potential and $K+$ homeostasis [22-24]. It has been shown that mice models that lacked the Slc26a4 gene $\left(S l c 26 a 4^{-/-}\right)$did not express Kcnj10 and that lack of this protein led to the loss of the endocochlear potential via endolymphatic acidification and $\mathrm{Ca}^{2+}$ absorption inhibition [25,26] which may be the direct cause of deafness in Pendred syndrome. Variants of KCNJ10 have also been considered as a risk factor for seizure susceptibility in genetic association studies [27] and biallelic mutations in KCNJ10 in humans are known to cause a syndromic form of hearing loss, EAST syndrome (epilepsy, ataxia, sensorineural deafness and renal tubulopathy) [28].

FOXI1 is a transcription factor gene and an upstream regulator of SCL26A4 [19,29]. It is also involved in the regulation of vascular $\mathrm{H}^{+}$-ATPase proton pumps in the inner ear, epididymis and kidney [30]. It was proposed that digenic inheritance of mutations in both FOXI1 and SLC26A4 were involved in the genetic basis of Pendred syndrome, as mutations in FOX1 were shown to reduce the transcription of SLC26A4 [19]. Moreover, Foxi1 null mice showed phenotypic features of sensorineural deafness due to the defective pendrin-chloride mediated reabsorption in which FOXI1 was most likely a key regulator [29].

Only a few studies have found evidence that KCNJ10 or FOXI1 gene mutations may influence the disease phenotype shown in patients with monoallelic mutations in the SLC26A4 gene. The present study investigates whether variants in KCNJ10 and FOXI1 in combination with heterozygosity for SLC26A4 mutations are likely to be associated with the disease phenotype seen in Pendred syndrome/EVA patients (who already have one mutation in the SLC26A4 gene). Because of the suggested role of KCNJ1O in both epilepsy and PDS/EVA, we also screened 3 unrelated patients with the combination of Pendred syndrome and seizures.

\section{Methods}

\section{Clinical and molecular evaluation of subjects}

68 patient samples were referred for screening of SLC26A4 because of a history of hearing impairment and either goitre or EVA. Available clinical details are given in Additional file 1. All patients gave informed consent for genetic testing for diagnosis of their deafness. All were referred for genetic testing by Clinical Geneticists, Genetic Counsellors or Paediatricians. A further 3 patients with Pendred syndrome (who had bi-allelic SLC26A4 mutations), who had a clinical history of seizures, were also screened for specific variants in KCNJ10/ FOXI1. In addition 95 pan-ethnic controls were sequenced for FOXI1.

\section{Genetic analyses}

Genomic DNA was extracted from whole blood to standard using Flexigene (Qiagen) chemistry on the Autogen Flex (Autogen) or, where samples were received from other NHS hospitals, were extracted using their normal protocols. Polymerase chain reaction (PCR) was performed on all samples with a total reaction volume of $20 \mu \mathrm{L}$ consisting of $0.5 \mu \mathrm{L}$ genomic DNA, $19 \mu \mathrm{L}$ Megamixmix (Microzone Ltd) and $0.5 \mu \mathrm{L}$ of forward and reverse tailed primers for each gene. All $3 \mathrm{KCNJ10}$ exons were amplified using the peltier thermal cycler (Biorad tetrad 2 DNA engine) and PCR was performed under the following PCR conditions: incubation at $95^{\circ} \mathrm{C}$ for 2 mins followed by 32 cycles of denaturation at $95^{\circ} \mathrm{C}$ for 30 seconds, annealing at $62^{\circ} \mathrm{C}$ for 30 seconds and an extension at $72^{\circ} \mathrm{C}$ for 30 seconds followed by a final 2 mins at $72^{\circ} \mathrm{C}$. All 4 FOXI1 exons were amplified with the same process but with the following touchdown PCR conditions: $95^{\circ} \mathrm{C}$ for 2 mins followed by $95^{\circ} \mathrm{C}$ for 30 seconds, $65^{\circ} \mathrm{C}$ for 30 seconds and $72^{\circ} \mathrm{C}$ for 30 seconds, decreasing the annealing temperature by $1^{\circ} \mathrm{C}$ for a further 14 cycles then $95^{\circ} \mathrm{C}$ for 30 seconds, $50^{\circ} \mathrm{C}$ for 30 seconds and $72^{\circ} \mathrm{C}$ for 30 seconds for a further 19 cycles followed by a final 2 mins at $72^{\circ} \mathrm{C}$.

\section{Primers}

Primers were designed using bioinformatics software, Primer3 (v4) (http://primer3.wi.mit.edu/). No SNPs were found using SNPCheckV3 (https://ngrl.manchester.ac. uk/SNPCheckV3/snpcheck.htm;jsessionid=1D413B67748 713D5A68E85D95C00D87C, NGRL Manchester). 
All primers had a tailed sequence added for automated Sanger sequencing. All forward and reverse primers were tailed at the $5^{\prime}$ of the primer (Table 1 ).

\section{Sequencing of PCR products}

The complete coding regions and splice sites of the KCNJ10 and FOXI1 exons were subjected to bidirectional sequencing using ABI PRISM Big Dye Terminator cycle sequencing ready reaction kit (Applied Biosystems) and analysed on an ABI3730 Genetic Analyser (Applied Biosystems) using standard protocols. The data was collated using Foundation Data Collection v3.0 and were analysed using the software program Mutation Surveyor v.3.30 (Softgenetics) and in comparison to the GenBank sequences of KCNJ10 (Genomic reference: NM_002241.4) and FOXI1 (Genomic reference: NM_012188.4). The data was also analysed for reported variants within the gene by using Alamut (Interactive Biosoftware).

\section{Results}

Screening of FOXI1 and KCNJ10 was completed for the cohort of 68 patients all of whom had previously been found to have only a single mutation in the SLC26A4 gene. Results are shown in Table 2. In KCNJ10, three unrelated patients were identified with the variant p.Arg271Cys (c.811C > T) and one with p.Arg18Gln (c.52G > A). In FOXI1, only one patient was observed to have a variant, p.Arg123Trp (c.367C > T). This variant was not found in the 95 pan-ethnic controls. No other mutations in either KCNJ10 or FOXI1 were found.

Results of the analysis of the three further patients referred for SLC26A4 screening who also had a history of seizures are shown in Table 3. One patient was found to have p.Arg271Cys, and one had the variant p.Arg18Gln.

\section{Discussion}

The evidence that genetic factors in addition to mutations in the coding region of the SLC26A4 gene contribute to Pendred syndrome and non-syndromic EVA has originated from both mouse and human studies. In humans the most compelling fact is that bi-allelic SLC26A4 mutations are found in only a minority of patients, even those with the most severe phenotype of EVA-Mondini and thyroid dysfunction or goitre. Undiscovered non-coding region mutations of $S L C 26 A 4$ are unlikely to fully explain this observation as a number of groups have performed extensive analysis of non-coding regions with little yield, although a variant in the promoter region of SLC26A4 which binds the transcription factor, FOXI1 was noted. Furthermore, siblings with discordant phenotypes, a mono-allelic SLC26A4 mutation and concordant SLC26A4 haplotypes, as well as siblings with concordant phenotypes but discordant haplotypes, indicate evidence for locus heterogeneity [9].

\section{Studies based on mouse models}

Studies in mice have suggested other genes that might influence the PDS/EVA phenotype and may act in a digenic, or additive manner. Mice lacking FOXI1 are deaf, have inner ear malformations (EVA and cochlear dysplasia) as well as renal tubular acidosis and male infertility. Furthermore these mice show no expression of SLC26A4 in the inner ear, implying that as an upstream regulator, FoxI1 expression is necessary for the transcription of Slc26a4. Therefore the contribution of FOXI1 to EVA/PDS was investigated in humans by Yang et al., who found a variant in the promoter of SLC26A4 in a consensus binding motif for FOXI1, c. $-101 \mathrm{~T}>\mathrm{C}$; they showed using EMSAs that binding affinity of FOXI1 to the promoter was reduced in the presence of c.-101T $>\mathrm{C}$ and used Luciferase reporter constructs to show that transcription from the promoter was completely abolished in the presence of this variant [19]. Further screening of the coding region of FOXI1 itself revealed five further non-synonymous variants, p. Asp161del, p. Arg267Gln, p.Gly335Val, p.Gly258Arg, p.Gly258Glu among 372 probands with EVA in whom one or zero SLC26A4 mutations had previously been found. All five mutations showed reduced activation of the luciferase reporter construct. Finally mice which are double

Table 1 Primers used to screen KCNJ10 and FOXI1

\begin{tabular}{|c|c|c|c|}
\hline Gene & Exon & Forward primer & Reverse primer \\
\hline \multirow[t]{3}{*}{ KCNJ10 } & $2 A$ & GGCACATAGCAAGTGCTCAA & AGATTTCCAGGATGGTGGTG \\
\hline & $2 B$ & TGGTGTGGTATCTGGTAGCTG & CAAAGTCACCCTCACCACTG \\
\hline & $2 \mathrm{C}$ & ACCCCTTACCTTCTATCATG & GTAGTATTCCTTACCAGGGC \\
\hline \multirow[t]{4}{*}{ FOXI1 } & $1 \mathrm{~A}$ & GCCAAGCCCTAGGGGTATAA & AGCTTCATCAGCTCCTCCTG \\
\hline & 1B & CAGGCCTATGGAGTGCAGA & CCATGCTCTCCTTCACTGCT \\
\hline & $2 \mathrm{~A}$ & GCCTGTCGGTCTCTGTCTTC & CTCAGTCCTGGTGTGACCAA \\
\hline & $2 \mathrm{C}$ & CATCTTGGATGGAGCCTCAC & CCTGCTTATGTCTGGGCAGT \\
\hline Tail & & AGCTAAGCGCGAGAAGGC & AGCTAAGCGCGAGAAGGC \\
\hline
\end{tabular}


Table 2 KCNJ10 and FOXI1 mutations identified in patients with monoallelic mutations in SLC26A4

\begin{tabular}{|c|c|c|c|c|}
\hline Patient & SLC26A4 mutation (cDNA) & SLC26A4 mutation (protein) & FOXI1 Variant & KCNJ10 Variant \\
\hline 25215 & c. $1001+1 G>A$ & & $\mathrm{~N}$ & $\mathrm{~N}$ \\
\hline 25278 & c. $1790 \mathrm{~T}>\mathrm{C}$ & p.Leu597Ser & N & N \\
\hline 34515 & c.716T > A & p. Val239Asp & N & $\mathrm{N}$ \\
\hline 28979 & C. $1061 T>C$ & p.Phe354Ser & N & N \\
\hline 35265 & c.707T $>C$ & p.Leu236Pro & N & N \\
\hline 40257 & $c .1001+1 G>A$ & & N & $\mathrm{N}$ \\
\hline 37952 & c. $1151 A>G$ & p.Glu384Gly & N & $\mathrm{N}$ \\
\hline 38201 & c. $1790 \mathrm{~T}>\mathrm{C}$ & p.Leu597Ser & N & $\mathrm{N}$ \\
\hline 42836 & c.707T >C & p.Leu236Pro & $\mathrm{N}$ & $\mathrm{N}$ \\
\hline 42564 & (c.1234G $>$ A & p.Val412lle) & $\mathrm{N}$ & $\mathrm{N}$ \\
\hline 41066 & C. $1001+1 G>A$ & & N & N \\
\hline 40187 & c. $412 \mathrm{G}>\mathrm{T}$ & p.Val138Phe & N & $\mathrm{N}$ \\
\hline 44595 & c.2127delT & p.Phe709Leuf* $* 12$ & N & c.811C > T (p.Arg271Cys)/N \\
\hline 45381 & c. $1790 \mathrm{~T}>\mathrm{C}$ & p.Leu597Ser & $\mathrm{N}$ & $\mathrm{N}$ \\
\hline 13343 & c.1342-2_1343dup & p.Leu450Glyfs*19 & N & $\mathrm{N}$ \\
\hline 45592 & c. $340 \mathrm{G}>\mathrm{A}$ & p.Gly114Arg & $\mathrm{N}$ & $\mathrm{N}$ \\
\hline 48799 & $c .1001+1 G>A$ & & N & N \\
\hline 46182 & c.707T >C & p.Leu236Pro & $\mathrm{N}$ & $\mathrm{N}$ \\
\hline 50939 & c. $2190 G>T$ & p.Gln730His & N & $\mathrm{N}$ \\
\hline 51079 & c.707T >C & p.Leu236Pro & N & $\mathrm{N}$ \\
\hline 54165 & $\mathrm{C} .2 \mathrm{~T}>\mathrm{C}$ & p.Met1? & $\mathrm{N}$ & $\mathrm{N}$ \\
\hline 59858 & c. $2080 T>C$ & p.Ser694Pro & N & $\mathrm{N}$ \\
\hline 61452 & c. $412 \mathrm{G}>\mathrm{T}$ & p.Val138Phe & $\mathrm{N}$ & $\mathrm{N}$ \\
\hline 54483 & C. $1790 \mathrm{~T}>\mathrm{C}$ & p.Leu597Ser & N & N \\
\hline 63420 & c. $1151 A>G$ & p.Glu384Gly & N & $\mathrm{N}$ \\
\hline 65983 & c. $1211 C>T$ & p.Thr404lle & $\mathrm{N}$ & $\mathrm{N}$ \\
\hline 50886 & c. $1151 A>G$ & p.Glu384Gly & $\mathrm{N}$ & $\mathrm{N}$ \\
\hline 66609 & c. $-3-2 A>G$ & & N & $\mathrm{N}$ \\
\hline 66643 & c. $[1001+1 G>A(;) 2219 C>T]$ & (p.Gly740Val) & N & $\mathrm{N}$ \\
\hline 66830 & c. $1790 \mathrm{~T}>\mathrm{C}$ & p.Leu597Ser & N & $\mathrm{N}$ \\
\hline 69863 & C. $113 T>C$ & p.Phe335Leu & $\mathrm{N}$ & $\mathrm{N}$ \\
\hline 47141 & c. $1790 \mathrm{~T}>\mathrm{C}$ & p.Leu597Ser & N & $\mathrm{N}$ \\
\hline 72446 & c. $-3-2 A>G$ & & N & N \\
\hline 72770 & $c .412 \mathrm{G}>\mathrm{T}$ & p.Val138Phe & N & $\mathrm{N}$ \\
\hline 72617 & c. $1151 A>G$ & p.Glu384Gly & N & $\mathrm{N}$ \\
\hline 76349 & c. $1790 \mathrm{~T}>\mathrm{C}$ & p.Leu597Ser & $\mathrm{N}$ & $\mathrm{N}$ \\
\hline 76715 & c. $1826 \mathrm{~T}>\mathrm{G}$ & p.Val609Gly & N & N \\
\hline 78124 & C. $1468 \mathrm{~A}>C$ & p.lle490Leu & N & $\mathrm{N}$ \\
\hline 78231 & $c .1001+1 G>A$ & & N & $\mathrm{N}$ \\
\hline 23853 & $\mathrm{C} .2 \mathrm{~T}>\mathrm{C}$ & p.Met1? & $\mathrm{N}$ & $\mathrm{N}$ \\
\hline 71753 & c.1342-2_1343dup & p.Leu450Glyfs*19 & N & $\mathrm{N}$ \\
\hline 72950 & c. $1229 C>T$ & p.Thr410Met & N & $\mathrm{N}$ \\
\hline 79945 & $(c .2219 C>T)$ & (p.Gly740Val) & c.367C > T(p.Arg123Trp) & $\mathrm{N}$ \\
\hline 80435 & c.707T > C & p.Leu236Pro & $\mathrm{N}$ & $\mathrm{N}$ \\
\hline
\end{tabular}


Table 2 KCNJ10 and FOXI1 mutations identified in patients with monoallelic mutations in SLC26A4 (Continued)

\begin{tabular}{|c|c|c|c|c|}
\hline 10576 & c. [1343C > T]; [1991C > T] & p.[Ser448Leu]; [Ala664Ser] & $\mathrm{N}$ & $\mathrm{N}$ \\
\hline 83112 & C. $1790 T>C$ & p.Leu597Ser & $\mathrm{N}$ & $\mathrm{N}$ \\
\hline 84175 & c.119delT (c.918G > A) & p.Leu40ArgfsX26 & $\mathrm{N}$ & $\mathrm{N}$ \\
\hline 86297 & c. $2153 G>T$ & p.Phe718Ser & $\mathrm{N}$ & c.53G > A $(p . \operatorname{Arg} 18 \mathrm{G} \ln ) / \mathrm{N}$ \\
\hline 86482 & c.707T >C & p.Leu236Pro & $\mathrm{N}$ & $\mathrm{N}$ \\
\hline 85020 & c. $1234 \mathrm{G}>\mathrm{T}$ & p.Gln421Arg & $\mathrm{N}$ & $\mathrm{N}$ \\
\hline 83883 & c. $1790 \mathrm{~T}>\mathrm{C}$ & p. Leu597Ser & $\mathrm{N}$ & $\mathrm{N}$ \\
\hline 87823 & C.1790T>C & p. Leu597Ser & $\mathrm{N}$ & $\mathrm{N}$ \\
\hline 84236 & $(c .73 C>T)$ & (p.Pro25Ser) & $\mathrm{N}$ & N \\
\hline 88933 & C. $1151 A>G$ & p.Glu384Gly & $\mathrm{N}$ & $\mathrm{N}$ \\
\hline 89770 & c. $1001+1 G>A$ & & $\mathrm{~N}$ & N \\
\hline 90473 & c. $1790 \mathrm{~T}>\mathrm{C}$ & p.Leu597Ser & $\mathrm{N}$ & c.811C > T (p.Arg271Cys) /N \\
\hline 90511 & c. $1003 \mathrm{~T}>\mathrm{C}$ & p.Phe335Leu & $\mathrm{N}$ & c.811C > T (p.Arg271Cys) /N \\
\hline 90643 & $(c .970 A>T)$ & (p.Asn324Tyr) & $\mathrm{N}$ & N \\
\hline 91820 & C.1790T >C & p.Leu597Ser & $\mathrm{N}$ & N \\
\hline 40013 & c. $1000 G>T$ & p.Gly334Trp & $\mathrm{N}$ & $\mathrm{N}$ \\
\hline 89620 & c.[1790T > C(;) 412G > T] & p.[Val138Phe(;) (Leu597Ser)] & $\mathrm{N}$ & N \\
\hline 94065 & c.1342-2_1343dup & p.Leu450Glyfs*19 & $\mathrm{N}$ & N \\
\hline 89792 & C.- $103 \mathrm{~T}>\mathrm{C}$ & & $\mathrm{N}$ & $N$ \\
\hline 94743 & c. $1003 T>C$ & p.Phe335Leu & $\mathrm{N}$ & $\mathrm{N}$ \\
\hline 96669 & c. $2015 G>A$ & p.Gly672Glu & $\mathrm{N}$ & $N$ \\
\hline 95020 & c. $1363 \mathrm{~A}>\mathrm{T}$ & p.lle455Phe & $\mathrm{N}$ & N \\
\hline 99311 & c.626G > T & p.Gly209Val & $\mathrm{N}$ & $\mathrm{N}$ \\
\hline 99458 & c. $1334 \mathrm{~T}>\mathrm{G}$ & p.Leu445Trp & $\mathrm{N}$ & $\mathrm{N}$ \\
\hline
\end{tabular}

Unclassified variants are denoted in brackets. p.Leu597Ser may be a benign polymorphism or hypofunctional variant [39].

heterozygotes for variants in Slc26a4 and Foxi1 $\left(S l c 26 a 4^{+/-}\right.$, Foxi1 ${ }^{+/-}$) were reported to have EVA although hearing of these mice was not commented on [19].

The potential role of the inwardly rectifying potassium channel KCNJ10 in PDS/EVA was also suggested from studies in mice. The Slc26a4 $4^{-1-}$ mouse is deaf and has EVA and shows loss of endocochlear potential and no expression of $\mathrm{KCNJ10}$ protein in the stria vascularis. $\mathrm{KCNJ10}$ is thought to be crucial in the generation and maintenance of the endocochlear potential necessary for auditory transduction. In 2 out of 89 EVA/PDS patients who were heterozygous for a single pathogenic SLC26A4 mutation, Yang et al. found two heterozygous mutations in KCNJ10 in unrelated probands, and suggested a probable digenic interaction between these two genes. In their hands, expression of mutant KCNJ10 in Xenopus oocytes showed markedly reduced $\mathrm{K}^{+}$conductance [21].

\section{Studies in humans}

However subsequent work by others has shown that recessive mutations in $\mathrm{KCNJ10}$ do indeed cause hearing loss, but as part of a complex syndrome, EAST syndrome, consisting of Epilepsy, Ataxia, Sensorineural deafness and renal Tubulopathy. Heterozygote parents are unaffected. In addition $K C N J 10$ has also been considered as a 'susceptibility gene' for epilepsy in humans. However the variant p.Arg271Cys was thought to be a protective allele against focal or generalised epilepsy $[22,31,32]$ and yet this was found in one of our patients with co-existing seizures and Pendred syndrome. As

Table 3 Patients with biallelic mutations of SLC26A4 tested for variants within the KCNJ10 gene

\begin{tabular}{lllll}
\hline Patient & SLC26A4 allele $\mathbf{1}$ & SLC26A4 allele 2 & KCNJ10 variant & dbSNP \\
\hline 66119 & c.1229C $>$ T p.Thr410Met & c.1229C $>$ T p.Thr410Met & c.811C > T p.Arg271Cys & rs1130183 \\
5472 & c.707T > C p.Leu236Pro & c.626G $>$ T p.Gly209Val & C.53G > A p.Arg18Gln & rs115466046 \\
6401 & c.707T > C p.Leu236Pro & c.707T > C p.Leu236Pro & $\mathrm{N}$ & \\
\hline
\end{tabular}


studies failed to show a functional or structural effect on channels, and because this variant is present in 563 of 8037 individuals (0.07) of European American descent in Exome Variant Server (http://evs.gs.washington. edu/EVS/), p.Arg271Cys is most likely to be a polymorphism, unrelated to either EVA/PDS or even epilepsy. Similarly, the variant p.Arg18Gln has also been reported previously, but in a single patient with autistic spectrum disorder, seizures and intellectual disability but no deafness [33]. This suggests that it may be unrelated to any of these phenotypes, consistent with its frequency in the population (present in 1 in 57 European Americans in EVS (148 in 8452 European Americans (0.0175)) (http:// evs.gs.washington.edu/EVS/). As the variant in FOXI1, p. Arg123Trp (c.367C > T) has not been found at a high frequency previously or in controls screened here, we cannot be sure whether this is, or is not, a pathogenic mutation. However it appears that mutations in FOXI1 are not a major contributor to digenic inheritance in these cases.

Other genetic studies of patients with EVA/PDS have failed to find convincing evidence that mutations of FOXI1 and KCNJ10 contribute to these phenotypes. Chen et al. [34] screened SLC26A4, FOXI1 and KCNJ10 (as well as GJB2) in patients with bilateral deafness and inner ear malformations and found no mutations in FOXI1 or KCNJ10 in the 15 who had one or zero SLC26A4 mutations; Mercer et al. [35] screened 51 patients with EVA and found no mutations in FOXI1 or KCNJ10; Jonard et al. [36] screened 25 patients with unilateral deafness and unilateral EVA but found no mutations in either KCNJ10 or FOXI1 and Wu et al. screened FOXI1 in 100 patients with EVA and found no mutations, although KCNJ10 was not screened at that time [37]. Cirello et al. did however find a novel missense variant in FOXI1 p.Pro239Leu, and 3 patients with the variant p. Arg271Cys in KCNJ10 out of 19 patients with PDS/EVA, but functional analysis of p.Pro239Leu showed no significant impairment in the transcriptional activation of SLC26A4, and, p.Arg271Cys was classified as polymorphism [38].

Our study is the largest study to screen both $K C N J 10$ and FOXI1 in patients who were heterozygous for a single SLC26A4 mutation in whom the promoter variant/FOXI1 (c.-101T > C) binding site had already been excluded. Our results indicate that heterozygosity for mutations in either of these genes in combination with a heterozygous SLC26A4 mutation is not a significant contributor to PDS/EVA in our cohort, even though previous in vitro work and mouse studies suggest their involvement in these phenotypes. The best evidence for a possible functional role of either KCNJ1O or FOXI1 in the PDS/EVA phenotype in humans would be genetic evidence - further families with mutations, which are shown to have a functional effect and which are present in a lower frequency in the general population than those with the PDS/EVA phenotype. Hopefully, NGS data from large sequencing efforts such as 1000 Genomes and EVS will be helpful in this respect.

It is possible that some of the cases presented here could be 'co-incidental' carriers for mutations in SLC26A4 which do not contribute to their phenotype of hearing loss, or that we could have missed single or multiple exon deletions. However we think that this is unlikely to account for a significant proportion of these cases. Renorff et al. found no deletions in their series of patients suggesting that deletions account for a very small number of mutations in SLC26A4 [39]. Regarding carrier ship, we have recently performed an audit of all requests for SLC26A4 screening performed in our laboratory from 2002 onwards. This shows that where testing of SLC26A4 was requested, 66 out of a total of 538 patients tested, had monoallelic mutations (ie. 1 in 8). Even if those with variants of unknown significance are excluded, the heterozygote carriership rate for SLC26A4 mutations would be almost 4 times that for GJB2, the commonest form of inherited deafness [40]. This suggests that heterozygosity for SLC26A4 mutations is important in the aetiology of the hearing loss in these patients.

\section{Conclusion}

This study combined with others, suggests that variants in KCNJ1O and FOXI1 are not a major contributor to PDS/ EVA and that screening of these genes in a diagnostic setting will add little information to genetic aetiology. Other factors, genetic or environmental, are likely to play a greater role in the aetiology of PDS/EVA.

\section{Additional file}

Additional file 1: Clinical Information on patients.

Competing interests

The authors declare no conflict of interest.

\section{Authors' contributions}

MBG and AMD designed the study and chose the patient cohort. PL performed the laboratory work under the supervision of AMD. MBG, AMD and $\mathrm{PL}$ all participated in the analysis of the results. PL wrote the initial manuscript which was edited and revised by MBG and AMD. KR and $L$ provided critical feedback and thoughts on the manuscript. All authors read and approved the final manuscript.

\section{Acknowledgements}

We would like to thank all the referring clinicians and their patients. MB-G is supported by Great Ormond Street Hospital Children's Charity.

\section{Author details}

${ }^{1}$ North East Thames Regional Genetics Service Laboratory, Great Ormond Street Hospital for Children NHS Foundation Trust, 37 Queen Square,York House, London WC1N 3BH, UK. ${ }^{2}$ Cochlear Implant Department, Great Ormond Street Hospital for Children NHS Foundation Trust, London WC1N $3 \mathrm{JH}$, UK. ${ }^{3}$ Clinical and Molecular Genetics Unit, UCL Institute of Child Health, 30 Guilford Street, London WC1N 1EH, UK. 
Received: 2 January 2013 Accepted: 6 August 2013

Published: 21 August 2013

\section{References}

1. Fraser GR: Association of congenital deafness with goitre (Pendred's syndrome). Ann Hum Genet 1965, 28:201-248.

2. Kopp P, Pesce L, Solis-S JC: Pendred syndrome and iodide transport in the thyroid. Trends Endocrinol Metab 2008, 19(7):260-268.

3. Pendred V: Deaf-mutism and goitre. 1896, 2:532.

4. Coyle B, Reardon W, Herbrick JA, Tsui LC, Gausden E, Lee J, et al: Molecular analysis of the PDS gene in Pendred syndrome. Hum Mol Genet 1998, 7(7):1105-1112.

5. Bizhanova A, Kopp P: Genetics and phenomics of Pendred syndrome. Mol Cell Endocrinol 2010, 322(1-2):83-90.

6. Phelps PD, Coffey RA, Trembath RC, Luxon LM, Grossman AB, Britton KE, et al: Radiological malformations of the ear in Pendred syndrome. Clin Radiol 1998, 53(4):268-273.

7. Yang JJ, Tsai CC, Hsu HM, Shiao JY, Su CC, Li SY: Hearing loss associated with enlarged vestibular aqueduct and Mondini dysplasia is caused by splice-site mutation in the PDS gene. Hear Res 2005, 199(1-2):22-30.

8. Joshua B, Kaplan DM, Raveh E, Lotan D, Anikster Y: Audiometric and imaging characteristics of distal renal tubular acidosis and deafness. J Laryngol Otol 2008, 122(2):193-198.

9. Pryor SP, Madeo AC, Reynolds JC, Sarlis NJ, Arnos KS, Nance WE, et al: SLC26A4/PDS genotype-phenotype correlation in hearing loss with enlargement of the vestibular aqueduct (EVA): evidence that Pendred syndrome and non-syndromic EVA are distinct clinical and genetic entities. J Med Genet 2005, 42(2):159-165.

10. Soleimani M, Greeley T, Petrovic S, Wang Z, Amlal H, Kopp P, et al: Pendrin: an apical Cl-/OH-I. Am J Physiol Renal Physiol 2001, 280(2):F356-F364.

11. Scott DA, Wang R, Kreman TM, Sheffield VC, Karniski LP: The Pendred syndrome gene encodes a chloride-iodide transport protein. Nat Genet 1999, 21(4):440-443.

12. TROTTER WR: The association of deafness with thyroid dysfunction. Br Med Bull 1960, 16:92-98.

13. Campbell C, Cucci RA, Prasad S, Green GE, Edeal JB, Galer CE, et al: Pendred syndrome, DFNB4, and PDS/SLC26A4 identification of eight novel mutations and possible genotype-phenotype correlations. Hum Mutat 2001, 17(5):403-411.

14. Everett LA, Glaser B, Beck JC, Idol JR, Buchs A, Heyman M, et al: Pendred syndrome is caused by mutations in a putative sulphate transporter gene (PDS). Nat Genet 1997, 17(4):411-422.

15. Coucke P, Van CG, Demirhan O, Kabakkaya Y, Balemans W, Van HP, et al: The gene for Pendred syndrome is located between D7S501 and D7S692 in a 1.7-cM region on chromosome 7q. Genomics 1997, 40(1):48-54.

16. Dai P, Stewart AK, Chebib F, Hsu A, Rozenfeld J, Huang D, et al: Distinct and novel SLC26A4/Pendrin mutations in Chinese and U.S. patients with nonsyndromic hearing loss. Physiol Genomics 2009, 38(3):281-290.

17. Li XC, Everett LA, Lalwani AK, Desmukh D, Friedman TB, Green ED, et al: A mutation in PDS causes non-syndromic recessive deafness. Nat Genet 1998, 18(3):215-217.

18. Glaser B: Pendred syndrome. Pediatr Endocrinol Rev 2003, 1(Suppl 2):199-204.

19. Yang T, Vidarsson H, Rodrigo-Blomqvist S, Rosengren SS, Enerback S, Smith $\mathrm{RJ}$ : Transcriptional control of SLC26A4 is involved in Pendred syndrome and nonsyndromic enlargement of vestibular aqueduct (DFNB4). Am J Hum Genet 2007, 80(6):1055-1063.

20. Azaiez H, Yang T, Prasad S, Sorensen JL, Nishimura CJ, Kimberling WJ, et al: Genotype-phenotype correlations for SLC26A4-related deafness. Hum Genet 2007, 122(5):451-457.

21. Yang T, Gurrola JG, Wu H, Chiu SM, Wangemann P, Snyder PM, et al: Mutations of KCNJ10 together with mutations of SLC26A4 cause digenic nonsyndromic hearing loss associated with enlarged vestibular aqueduct syndrome. Am J Hum Genet 2009, 84(5):651-657.

22. Shang L, Lucchese CJ, Haider S, Tucker SJ: Functional characterisation of missense variations in the Kir4.1 potassium channel (KCNJ10) associated with seizure susceptibility. Brain Res Mol Brain Res 2005, 139(1):178-183.

23. Hibino H, Horio $Y$, Inanobe A, Doi K, Ito M, Yamada M, et al: An ATPdependent inwardly rectifying potassium channel, KAB-2 (Kir4. 1), in cochlear stria vascularis of inner ear: its specific subcellular localization and correlation with the formation of endocochlear potential. J Neurosci 1997, 17(12):4711-4721.
24. Reimann F, Ashcroft FM: Inwardly rectifying potassium channels. Curr Opin Cell Biol 1999, 11(4):503-508.

25. Wangemann P, Nakaya K, Wu T, Maganti RJ, Itza EM, Sanneman JD, et al: Loss of cochlear HCO3-secretion causes deafness via endolymphatic acidification and inhibition of $\mathrm{Ca} 2+$ reabsorption in a Pendred syndrome mouse model. Am J Physiol Renal Physiol 2007, 292(5):F1345-F1353.

26. Wangemann P, Itza EM, Albrecht B, Wu T, Jabba SV, Maganti RJ, et al: Loss of KCNJ10 protein expression abolishes endocochlear potential and causes deafness in Pendred syndrome mouse model. BMC Med 2004, 2:30.

27. Ferraro TN, Golden GT, Smith GG, Martin JF, Lohoff FW, Gieringer TA, et al: Fine mapping of a seizure susceptibility locus on mouse chromosome 1: nomination of Kcnj10 as a causative gene. Mamm Genome 2004, 15(4):239-251.

28. Bockenhauer D, Feather S, Stanescu HC, Bandulik S, Zdebik AA, Reichold M, et al: Epilepsy, ataxia, sensorineural deafness, tubulopathy, and KCNJ10 mutations. N Engl J Med 2009, 360(19):1960-1970.

29. Hulander M, Kiernan AE, Blomqvist SR, Carlsson P, Samuelsson EJ, Johansson $B R$, et al: Lack of pendrin expression leads to deafness and expansion of the endolymphatic compartment in inner ears of Foxi1 null mutant mice. Development 2003, 130(9):2013-2025.

30. Vidarsson H, Westergren R, Heglind M, Blomqvist SR, Breton S, Enerback S: The forkhead transcription factor Foxi1 is a master regulator of vacuolar H-ATPase proton pump subunits in the inner ear, kidney and epididymis. PLoS One 2009, 4(2):e4471.

31. Lenzen KP, Heils A, Lorenz S, Hempelmann A, Hofels S, Lohoff FW, et al: Supportive evidence for an allelic association of the human KCNJ10 potassium channel gene with idiopathic generalized epilepsy. Epilepsy Res 2005, 63(2-3):113-118.

32. Buono RJ, Lohoff FW, Sander T, Sperling MR, O'Connor MJ, Dlugos DJ, et al: Association between variation in the human KCNJ10 potassium ion channel gene and seizure susceptibility. Epilepsy Res 2004, 58(2-3):175-183.

33. Sicca F, Imbrici P, D'Adamo MC, Moro F, Bonatti F, Brovedani P, et al: Autism with seizures and intellectual disability: possible causative role of gain-of-function of the inwardly-rectifying $\mathrm{K}+$ channel Kir4.1. Neurobiol Dis 2011, 43(1):239-247.

34. Chen K, Wang X, Sun L, Jiang H: Screening of SLC26A4, FOXI1, KCNJ10, and GJB2 in bilateral deafness patients with inner ear malformation. Otolaryngol Head Neck Surg 2012, 146(6):972-978.

35. Mercer S, Mutton P, Dahl HH: Identification of SLC26A4 mutations in patients with hearing loss and enlarged vestibular aqueduct using high-resolution melting curve analysis. Genet Test Mol Biomarkers 2011, 15(5):365-368.

36. Jonard L, Niasme-Grare M, Bonnet C, Feldmann D, Rouillon I, Loundon N, et al: Screening of SLC26A4, FOXI1 and KCNJ10 genes in unilateral hearing impairment with ipsilateral enlarged vestibular aqueduct. Int I Pediatr Otorhinolaryngol 2010, 74(9):1049-1053.

37. Wu CC, Lu YC, Chen PJ, Yeh PL, Su YN, Hwu WL, et al: Phenotypic analyses and mutation screening of the SLC26A4 and FOXI1 genes in 101 Taiwanese families with bilateral nonsyndromic enlarged vestibular aqueduct (DFNB4) or Pendred syndrome. Audiol Neurootol 2010, 15(1):57-66.

38. Cirello V, Bazzini C, Vezzoli V, Muzza M, Rodighiero S, Castorina P, et al: Molecular and functional studies of 4 candidate loci in Pendred syndrome and nonsyndromic hearing loss. Mol Cell Endocrinol 2012, 351(2):342-350.

39. Rendtorff N, Schrijver I, Lodahl M, Rodriguez-Paris J, Johnsen T, Hansen E, et al: SLC26A4 mutation frequency and spectrum in 109 Danish Pendred syndrome/DFNB4 probands and a report of nine novel mutations. Clin Genet 2013. doi:10.1111/cge.12074.

40. Hall A, Pembrey M, Lutman M, Steer C, Bitner-Glindzicz M: Prevalence and audiological features in carriers of GJB2 mutations, c.35delG and c.101T > C (p.M34T), in a UK population study. BMJ Open 2012, 2:e001238. doi:10.1136/bmjopen-2012-001238.

doi:10.1186/1471-2350-14-85

Cite this article as: Landa et al:: Lack of significant association between mutations of KCNJ10 or FOXI1 and SLC26A4 mutations in pendred syndrome/enlarged vestibular aqueducts. BMC Medical Genetics 2013 14:85 